\title{
Melanoma vulvovaginal com recidiva em vigência de tratamento clínico
}

\author{
Julia Ferro1; Laís Antunes de Lima²; Luiza Alves Nabarros³; Marinna \\ Poletto Rizzardo4; Luciane Donato5; Rodrigo Ughini Villarroel ${ }^{6}$.
}

\section{Resumo}

Introdução: menos de 1\% dos melanomas acometem a vulva, apesar disso $10 \%$ de todos os cânceres de vulva são melanomas. A clínica é ampla e o estadiamento varia do melanoma vulvar para o vaginal. O prognóstico é reservado quando comparado ao do melanoma cutâneo. Método: relata-se o caso de uma paciente de 64anos, branca e menopáusica há 11anos que procurou atendimento por sangramento vaginal. Refere intensa exposição solar durante 26anos. Ao exame especular viu-se pequena lesão vaginal que na biópsia evidenciou neoplasia de células redondas com imunohistoquímica comprovando melanoma. Realizou cirurgia excisional, tendo a patologia da peça evidenciado melanoma do tipo nodular, nível IV de Clark, Breslow $12 \mathrm{~mm}$, presença de ulceração, alto número de mitoses, margens livres e sem metástase linfonodal. Realizou tratamento com alfa-interferona e acompanhamento ambulatorial durante 6meses. Após esse período, teve episódios de sangramento vaginal, hematoquezia, diarreia, dor ao evacuar, astenia e emagrecimento. Ao exame, linfonodomegalia em região inguinal esquerda e exteriorização de lesão vegetante e friável no canal vaginal com invasão do reto por contiguidade. Tomografia mostrou recidiva vulvovaginal

\footnotetext{
${ }^{1}$ Acadêmica graduanda em Medicina pela Universidade de Passo Fundo. E-mail: j.decostaferro@gmail.com

${ }^{2}$ Acadêmica graduanda em Medicina pela Universidade de Passo Fundo. E-mail: al.lais@hotmail.com

${ }^{3}$ Acadêmica graduanda em Medicina pela Universidade de Passo Fundo. E-mail: luizanabarros@gmail.com

${ }^{4}$ Acadêmica graduanda em Medicina pela Universidade de Passo Fundo. E-mail: ninnarizzardo@gmail.com

${ }^{5}$ Médica residente do Serviço de Cancerologia Clínica do Hospital da Cidade de Passo Fundo. E-mail: lucianedonato1@gmail.com

${ }^{6}$ Médico Preceptor da Residência do Serviço de Cancerologia Clínica do Hospital da Cidade de Passo Fundo.E-mail: ughini@hotmail.com
} 
com infiltração da região retal distal, metástases pulmonares e linfonodomegalia para aórtica esquerda. A literatura demonstra que melanoma proveniente do trato urogenital feminino ocorre $95 \%$ das vezes na vulva e $3 \%$ na vagina. Podem ocorrer prurido, sangramento vaginal, leucorreia, dispareunia e presença de massa. O estadiamento do melanoma vulvar se faz pelo sistema TNM, sendo o tamanho avaliado pelo estadiamento de Clark e Breslow; já o dos vaginais ainda não tem sistema adequado, sendo avaliada a extensão do tumor. $\mathrm{O}$ tratamento inicial para $\mathrm{o}$ melanoma vulvovaginal é cirúrgico. Em tumores irressecáveis, considera-se a radioterapia combinada com quimioterapia e imunoterapia. A taxa de sobrevida em 5anos do melanoma vulvar é de 24 a 77\%, já do melanoma vaginal é de 5 a $25 \%$. Resultados: melanomas vulvovaginais são raros e ainda não têm estudos suficientes para estabelecer padrão adequado de diagnóstico, estadiamento e tratamento, oferecendo prognóstico reservado. Conclusão: pela raridade da doença e pela escassez de dados, reforça-se a necessidade da adesão das pacientes a estudos clínicos. Devido ao caráter agressivo da doença, é imprescindível que haja avanço nos tratamentos existentes, buscando melhorar a qualidade de vida das pacientes e o prognóstico da doença.

Palavras Chave: melanoma; vulvovaginal; recidiva; urogenital; sangramento. 